\title{
Association between Physician Intensity of Antibiotic Prescribing and the Prescription of Benzodiazepines, Opioids and Proton-Pump Inhibitors to Nursing Home Residents: a Population-Based Observational Study
}

\author{
Kieran L. Quinn, MD, MSc 1,2,3,4 , Michael A. Campitelli, $\mathrm{MPH}^{2}$, Christina Diong, $M S C^{2}$, \\ Nick Daneman, MD, MSc 1,2,3,5,6, Nathan M. Stall, MD ${ }^{3,7,8}$, Andrew M. Morris, MD, SM ${ }^{7,4,9}$, \\ Allan S. Detsky, MD, $P h D^{1,3,4}$, Lianne Jeffs, RN, PhD, FAAN ${ }^{3,10}$, Colleen J. Maxwell, PhD 2, 11 , \\ Chaim M. Bell, MD, $P h D^{1,2,3,4}$, and Susan E. Bronskill, $P h D^{2,3,5,7}$
}

\begin{abstract}
'Department of Medicine, University of Toronto, Toronto, ON, Canada; ${ }^{2}$ ICES, Toronto, ON, Canada; ${ }^{3}$ Institute of Health Policy, Management and Evaluation, University of Toronto, Toronto, ON, Canada; ${ }^{4}$ Department of Medicine, Sinai Health System, Toronto, Ontario, Canada; ${ }^{5}$ Sunnybrook Research Institute, Toronto, ON, Canada; 'Division of Infectious Diseases, Sunnybrook Health Sciences Centre, University of Toronto, Toronto, ON, Canada; ${ }^{2}$ Women's College Research Institute, Women's College Hospital, Toronto, ON, Canada; ${ }^{8}$ Division of Geriatric Medicine, University of Toronto, Toronto, ON, Canada; 'Department of Medicine, University Health Network, Toronto, ON, Canada; ${ }^{10}$ St. Michael's Hospital, Toronto, ON, Canada; ${ }^{11}$ Schools of Pharmacy and Public Health \& Health Systems, University of Waterloo, Waterloo, ON, Canada.
\end{abstract}

\begin{abstract}
BACKGROUND: Prescribing patterns for episodic medications, such as antibiotics, might make useful surrogate measures of a physician's overall prescribing practice because use is common, and variation exists across prescribers. However, the extent to which a physician's current antibiotic prescribing practices are associated with the rate of prescription of other potentially harmful medications remains unknown.
\end{abstract}

OBJECTIVE: To examine the association between a physician's rate of antibiotic prescribing and their prescribing rate of benzodiazepines, opioids and proton-pump inhibitors in older adults.

DESIGN: Population-based cohort study in nursing homes in Ontario, Canada, which provides comprehensive clinical, behavioural and functional information on all patients.

PARTICIPANTS: 1926 physicians who provided care among 128,979 physician-patient pairs in 2015.

MAIN MEASURES: Likelihood of prescribing a benzodiazepine, opioid or proton-pump inhibitor between low-, average- and high-intensity antibiotic prescribers, adjusted for patient characteristics.

KEY RESULTS: Compared with average-intensity antibiotic prescribers, high-intensity prescribers had an increased likelihood of prescribing a benzodiazepine (odds ratio 1.21 [95\% CI, 1.11-1.32]), an opioid (odds ratio 1.28 [95\% CI, 1.17-1.39]) or a proton-pump inhibitor (odds ratio 1.38 [95\% CI, 1.27-1.51]]. High-intensity antibiotic prescribers were more likely to be high prescribers of all three medications (odds ratio 6.24 [95\% CI, 2.90-13.39])

Electronic supplementary material The online version of this article (https://doi.org/10.1007/s11606-019-05333-8) contains supplementary material, which is available to authorized users.

Received January 8, 2019

Revised July 11, 2019

Accepted August 21, 2019

Published online October 1, 2019 and also more likely to initiate all three medications, compared with average-intensity prescribers.

CONCLUSIONS: The intensity of a physician's episodic antibiotic prescribing was significantly associated with the likelihood of new and continued prescribing of opioids, benzodiazepines and proton-pump inhibitors in nursing homes. Patterns of episodic prescribing may be a useful mechanism to target physician-level interventions to optimize general prescribing behaviors, instead of prescribing behaviors for single medications.

KEY WORDS: physicians practice patterns; drug prescriptions; antibacterial agents; inappropriate prescribing; nursing home.

J Gen Intern Med 34(12):2763-71

DOI: $10.1007 / \mathrm{s} 11606-019-05333-8$

(c) Society of General Internal Medicine 2019

\section{INTRODUCTION}

Opioids, benzodiazepines and proton-pump inhibitors have been highlighted by several initiatives ${ }^{1-4}$ as targets for prescribing practice interventions because these medications are commonly prescribed and associated with risks of fall, fracture and death. ${ }^{5-10}$ Consequently, many jurisdictions identify physicians who are outliers in the prescribing of single medication classes in an attempt to reduce potentially inappropriate use and associated outcomes. ${ }^{111-13}$ Focus on a single medication class may be inefficient in changing a physician's prescribing practice more broadly, particularly if the focus is on curbing the prescribing of multiple medications simultaneously. ${ }^{14} \mathrm{De}-$ termining the responsibility of individual physicians for prescribing decisions, such as initiating a new medication or continuing a previous prescription from another physician, can be difficult, especially for multiple chronic medications where many different prescribers may be involved. For 
example, patients who receive ongoing prescriptions for opioids, ${ }^{15,16}$ benzodiazepines ${ }^{17,18}$ and proton-pump inhibitors over long periods of time may have those prescriptions newly started by one physician, and then renewed by many different physicians for a variety of reasons like inertia, making it difficult to determine who is really responsible for that behavior.

One solution to this problem may be to demonstrate that overprescribing of single episodic medications, such as antibiotics, is associated with the prescribing of multiple other chronically prescribed medications. Episodic medications are a good choice for this purpose because of the prevalence and variation in their use between physicians, ${ }^{19,20}$ and because it is easy to identify the physician responsible for prescribing them. This approach offers an advantage over the one that identifies high prescribers of medications used chronically, where multiple prescribers are often involved, which makes it difficult to ascribe responsibility to an individual physician.

The extent to which a physician's current antibiotic prescribing practice is associated with the prescription of multiple other chronically prescribed medications remains unknown. This study measured the association between a physician's rate of antibiotic prescribing and the likelihood that they prescribed benzodiazepines, opioids, proton-pump inhibitors (PPIs) or all three medications concurrently in nursing homes.

\section{METHODS}

\section{Study Design, Setting and Data Sources}

We conducted a population-based cohort study in Ontario, Canada, using linked clinical and health administrative databases. Ontario is Canada's most populous province with over 13 million residents. The administrative datasets used in this study were linked using encoded identifiers at the individual patient level and analyzed at ICES. ICES was previously known as the Institute for Clinical Evaluative Sciences, but recently changed its name to simply "ICES" (see https://www.ices.on.ca/About-ICES/Newname-and-logo). ICES is an independent, non-profit research institute funded by an annual grant from the Ontario Ministry of Health and Long-Term Care (MOHLTC). These databases have routinely been used to conduct pharmacoepidemiologic studies, ${ }^{21,22}$ including research on antibiotic prescribing in older adults (Online Supplement). ${ }^{20,23,24}$ In Ontario, all adults aged $\geq 65$ years have universal access to hospital care, medically necessary physicians' services and prescription drug insurance coverage.

The use of data in this project was authorized under Section 45 of Ontario's Personal Health Information Protection Act, which does not require review by a Research Ethics Board.

\section{Study Cohort}

We created a set of physician-patient prescribing pairs by identifying all 7632 physicians who prescribed at least one medication to a patient in a nursing home between January 1 and December 31, 2015. In Canada, patients in most nursing homes are cared for by an individual physician assigned to that nursing home who is not typically their previous primary care provider. Patients aged 66 years and older were chosen to allow for one complete year of historical medication claims.

The nursing home setting was intentionally chosen for two reasons. First, these patients represent a particularly vulnerable population of older persons known to be frequently exposed to potentially inappropriate medications. ${ }^{23,25,26}$ Second, we used the nursing home setting as an experimental model to reflect prescribing behaviors. The comprehensive clinical, behavioural and functional information routinely collected for all patients in Ontario nursing homes through the Resident Assessment Instrument (RAI) version $2.0^{27}$ allowed our study to adjust for several important cognitive and functional patientspecific factors that may confound the association, ${ }^{23}$ which are not available in traditional administrative data for communitydwelling adults.

Physicians with fewer than 100 medication prescriptions over the year were excluded because we did not wish to identify prescribing practices of those physicians who may be providing "on-call" coverage for a patient's longitudinal physician. Physicians were required to have certification in Family Medicine, Internal Medicine or Geriatric Medicine in order to increase the likelihood that they represented a patient's primary physician (and responsible for prescribing antibiotics in the nursing home setting). Patients were excluded if they did not have a completed full RAI assessment in the years 2014 and 2015. Figure 1 illustrates that our final cohort included 1926 physicians paired with 94,285 unique patients. Patients could be included in the physician-patient pairs more than once because a patient could be paired with multiple physicians. However, the individual physician responsible for prescribing a medication to their patient was identified through the medication claims database. Seventy-two percent of patients paired with only 1 physician, $22 \%$ with 2 physicians, $5 \%$ with 3 physicians and $1 \%$ with $\geq 4$ physicians, thereby creating a total sample of 128,979 physician-patient pairs.

\section{Exposure: Variability and Intensity of Antibiotic Prescribing}

Similar to our prior research, ${ }^{20}$ a physician's antibiotic prescribing intensity was calculated based on the proportion of patients to whom they prescribed an antibiotic, among all patients to whom they prescribed at least one medication in 2015. Funnel plots determined whether the between-physician variation in the intensity of antibiotic prescribing was greater than that expected by random chance (eFigure 1 online). ${ }^{28}$ Physicians were categorized as "low" (below the lower 2 


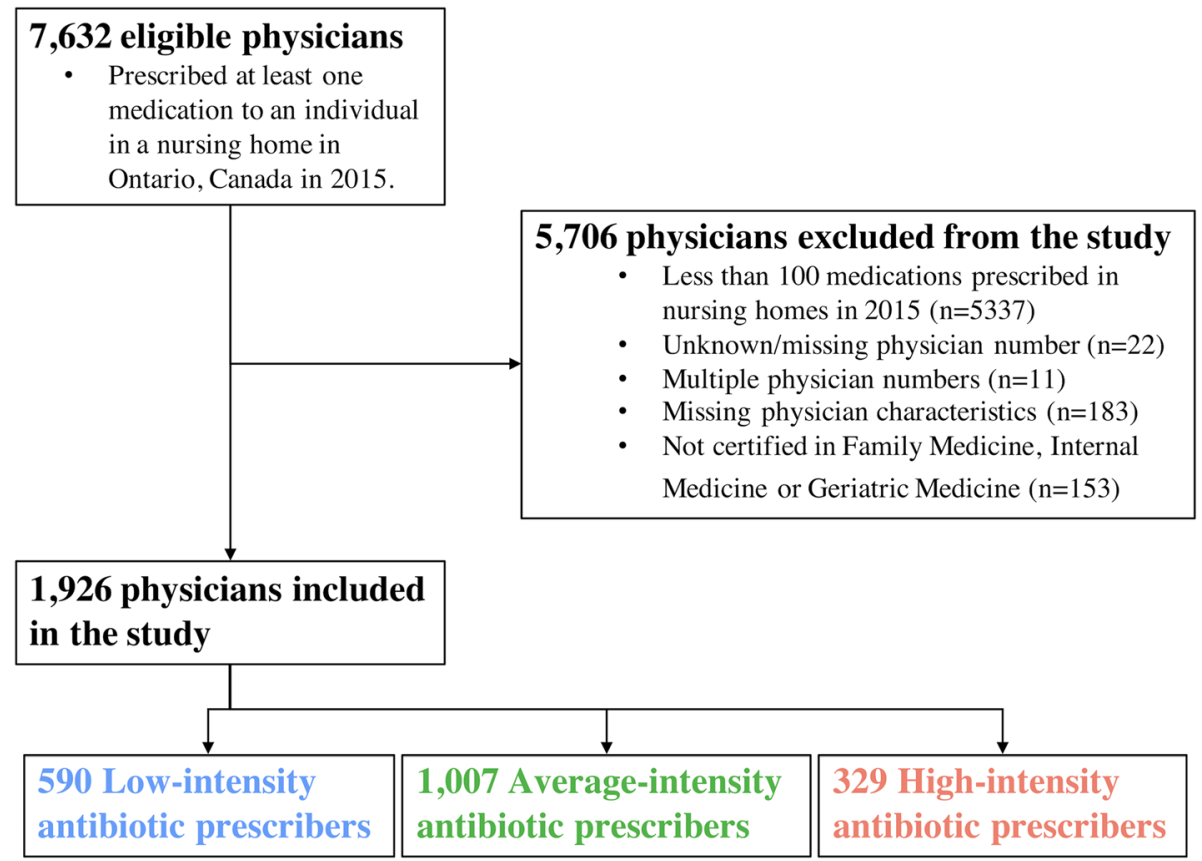

Figure 1 Flow diagram for creation of study cohort. All physicians who concurrently provided care in nursing homes in Ontario, Canada, were assessed for inclusion in the study. Family physicians, geriatricians and general internists who prescribed at least 100 medications annually to patients in $\mathbf{2 0 1 5}$ were included and subsequently stratified according to their intensity of antibiotic prescribing using funnel plot methodology.

standard deviation (SD) limit)-, "high" (above the upper 2 SD limit)- and "average" (all others)-intensity physicians based on their overall antibiotic prescribing intensity.

Based on this analysis, the median proportion of patients who received an antibiotic among the physician-patient pairs was $42 \%$ (IQR $0.24-0.53$ ). The numbers of physicians classified as low-, average-, or high-intensity antibiotics prescribers were $590(30.6 \%), 1007(52.3 \%)$ and $329(17.1 \%)$, respectively (eFigure 1 online).

\section{Outcome: Physician Likelihood of Prescribing Benzodiazepines, Opioids and Proton-Pump Inhibitors}

The primary outcomes were the likelihood of a physician prescribing a benzodiazepine, opioid or PPI to a paired patient in 2015. We chose these medications because (a) they are commonly prescribed and carry a risk of harm; ${ }^{15}$ (b) the American Geriatrics Society lists these medications as potentially inappropriate for use in older adults ${ }^{29}$ and (c), in the case of opioids specifically, prior research demonstrates the risk of long-term use for patients is associated with an individual physician and their prescribing tendencies. $^{30}$

In a secondary pre-specified analysis, we compared physicians across different antibiotic prescribing intensity groups and determined if they were also high-intensity prescribers (using funnel plots) of one or more of benzodiazepines, opioids and PPIs. A physician was assigned 1 point for each class of medication in which they were a high-intensity prescriber, corresponding to a maximum prescribing score of 3 . Each group was mutually exclusive, meaning that an individual physician could not have more than one prescribing score.

We performed two tracer analyses using levothyroxine and $\beta$-blocker ophthalmic drops as the outcome to test if highintensity prescribers modeled this behavior across all medication classes, or if they were limited to our specific medications of interest. We selected these medications because they are commonly prescribed, and we anticipated no association with antibiotic prescribing intensity due to their narrow indications for use. ${ }^{31}$ Levothyroxine is indicated only in the treatment of biochemically confirmed hypothyroidism. $\beta$-Blocker ophthalmic drops represent a unique group of medications that often require objective measurement of intraocular pressure, and are largely prescribed by ophthalmologists. ${ }^{31}$ Therefore, we expected prescribing of this medication by a patient's primary care physician to exclusively represent a continuation of specialist prescribing and to be independent of their antibiotic prescribing intensity.

\section{Patient and Physician Characteristics}

Patient characteristics included demographic, clinical and functional variables including length of stay in the nursing home, healthcare use in the prior year, comorbidities, functional dependence, cognitive impairment, aggressive behavior and device use such as indwelling catheters and feeding tubes. $^{23,24}$ Additionally, assessment items from the RAI 2.0 were used to derive a modified version of a previously validated frailty index. ${ }^{25,32,33}$

We also determined several physician-level characteristics, including age, sex, graduation from a Canadian versus foreign medical school, clinical specialty, number of years in practice, 
rural practice setting and the volume of long-term care and non-long-term care physician service fee claims during the study time frame.

\section{Statistical Analysis}

Multivariable, hierarchical logistic regression was used to measure the association between a physician's antibiotic prescribing intensity and the prescription of a benzodiazepine, opioid or PPI, accounting for patient characteristics (age, sex, emergency department visits and hospitalizations, and comorbidities such as including diabetes, congestive heart failure, hypertension, atherosclerotic heart disease, stroke, peripheral vascular disease, dementia, cancer, chronic obstructive pulmonary disease, Parkinson's disease, gastrointestinal disease, liver disease, renal failure, depression and anxiety, devices such as urinary catheters, frailty and functional status using the RAI scales) and the clustering of physician-patient pairs within physicians. The models also included the total number of unique medications dispensed in the previous year to control for the possibility that antibiotic prescribing practices were correlated with polypharmacy in any given patient.

We used multinomial logistic regression in our secondary analysis to estimate the association between a physician's antibiotic prescribing intensity level and the total number of medication classes (benzodiazepines, opioids and PPIs) in which they were a high-intensity prescriber. We compared high- with average-intensity antibiotic prescribers across the different prescriber scores. A score of 0 served as the reference for comparison.
A pre-specified sensitivity analysis was conducted examining new use of the study medications to minimize the possibility that prescribers were continuing chronic medications initiated by a different physician; incident medication use was determined using a 1-year lookback window for prior use.

All analyses were performed using SAS version 9.3 (SAS Institute, Cary, NC) and used a two-sided type 1 error rate of 0.05 as the threshold for statistical significance.

\section{RESULTS}

\section{Characteristics of Prescribing Physicians and the Variation in Their Prescribing of Benzodiazepines, Opioids and Proton-Pump Inhibitors}

Among our cohort of 1926 physicians, the majority were Canadian-trained, middle-aged males who practiced in an urban setting with over 25 years of experience (eTable 1 online). However, physicians who were high-intensity antibiotic prescribers were more likely to be male and international medical graduates practicing in an urban setting. They also provided care for more patients, more often, and had a higher proportion of total and nursing home medication and physician billing claims than average- and low-intensity prescribers. There was, however, significant variation within each group. Most physicians prescribed at least one of the study medications to at least one patient during the study period.

Figure 2 illustrates that there was a wide variation in the unadjusted use of benzodiazepines, opioids and PPIs across

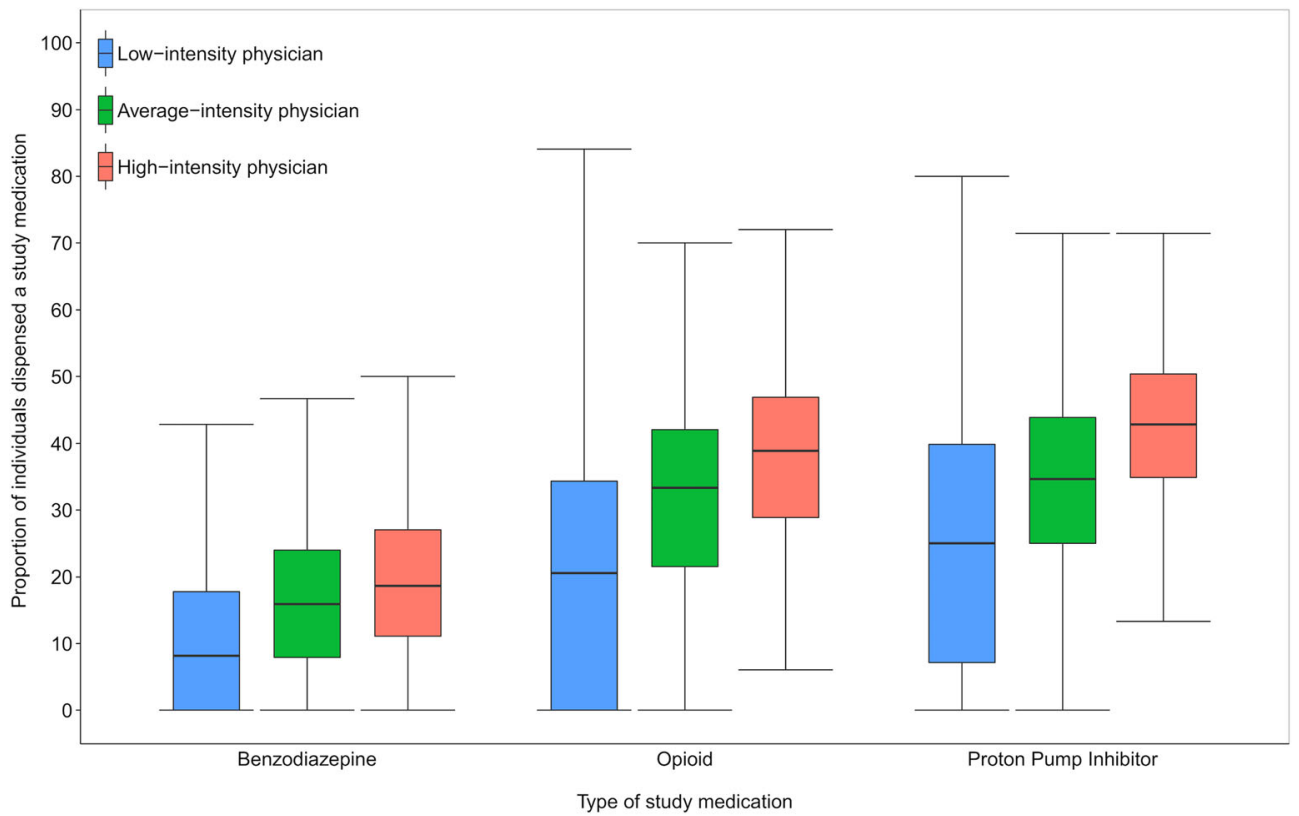

Figure 2 Box plot of the variation in proportions of study medication use among physician-patient pairs across prescribing physicians in Ontario 2015, by physician antibiotic prescribing intensity. Physicians were grouped according to their antibiotic prescribing intensity and paired with the patients to whom they prescribed medications in 2015. There was wide variation observed in the use of benzodiazepines, opioids and proton-pump inhibitors, and high-intensity antibiotic prescribers demonstrated higher use for all three compared with average- and lowintensity prescribers. 
antibiotic intensity groups, with each study medication being used more commonly among high-intensity antibiotic prescribers. The median proportion of patients who received a benzodiazepine between low-, average- and high-intensity antibiotic prescribers was $21 \%$ (interquartile range (IQR) 0 34), 33\% (IQR 21-42) and 39\% (IQR 29-47), respectively. Similarly, the proportions for opioids were 8\% (IQR 0-18), 16\% (IQR 8-24) and 19\% (IQR 11-27), and for PPIs were 25\% (IQR 7-40), 35\% (IQR 25-44) and 43\% (IQR 35-50). $\beta$ Blocker eye drops were rarely used, but unexpectedly, prescribing of both $\beta$-blocker eye drops and levothyroxine followed a similar gradient of increasing use across the different antibiotic intensity groups (eTable 1 online).

\section{Individual Patient Characteristics}

Overall, the sociodemographic and clinical characteristics of patients treated by high-intensity antibiotic prescribers were very similar to those treated by average- and low-intensity prescribers (eTable 2 online). Patients were typically older females with multiple comorbidities who were also frail. They were cumulatively prescribed a median of 16 different medications over the year. Alzheimer's and related dementias were common and approximately $20 \%$ of patients had severe cognitive impairment. Most patients required extensive assistance with or were totally dependent for their activities of daily living. A minority suffered with daily pain.

The proportions of patients who were receiving benzodiazepines, opioids and PPIs across the different groups of antibiotic prescribing intensity reflected the same prescribing gradient that was seen at the physician level (Table 1).

\section{Physician Antibiotic Prescribing Intensity and Likelihood of Prescribing Benzodiazepines, Opioids, Proton-Pump Inhibitors, Levothyroxine and $\beta$-Blocker Ophthalmic Drops}

The likelihood of a physician prescribing a benzodiazepine, opioid or PPI was higher among high-intensity antibiotic physicians compared with average-intensity physicians, after adjusting for patient factors (odds ratio 1.21 [95\% CI, 1.111.32 ]; 1.28 [95\% CI, 1.17-1.39] and 1.38 [95\% CI, 1.27-1.51] respectively, $p<0.001$ ) (Table 2).

Our tracer analysis also identified an association between high-intensity antibiotic prescribers and the likelihood of prescribing levothyroxine (adjusted odds ratio 1.23 [95\% CI, 1.16-1.31], $p<0.001)$ and $\beta$-blocker ophthalmic drop (adjusted odds ratio 1.10 [95\% CI, 1.02-1.19], $p=$ 0.01) (Table 2).

\section{Association Across Physician-Level Rates of Prescribing Benzodiazepines, Opioids and Proton-Pump Inhibitors}

Fifty seven percent of high-intensity antibiotic prescribers were also high prescribers of other medications (34.7\% for one, $17.9 \%$ for two, $4.6 \%$ for all three medications, respectively). High-intensity prescribers of antibiotics were significantly more likely than averageintensity prescribers to prescribe one (odds ratio 3.35, 95\% CI 2.49-4.50), two (odds ratio 6.64, 95\% CI 4.3610.12 ) or all three (odds ratio $6.24,95 \%$ CI $2.90-13.39$ ) $(p<0.001$ for all three) medications at a high intensity (Table 3).

\section{Physician Antibiotic Prescribing Intensity and Likelihood of Newly Prescribing Benzodiazepines, Opioids and Proton-Pump Inhibitors}

The likelihood of initiating a new prescription for a benzodiazepine, opioid or PPI remained higher among high-intensity antibiotic physicians than among average-intensity physicians, after adjusting for patient factors (odds ratio 1.22 [95\% CI, 1.12-1.34], $p<0.001 ; 1.26$ [95\% CI, 1.16-1.36], $p<0.001$; and 1.20 [95\% CI, 1.10-1.30], $p<0.001$, respectively). The same pattern was observed for the prescription of levothyroxine and $\beta$-blocker eye drops (odds ratio 1.17 [95\% CI, 1.08-1.28], $p<0.001 ; 1.19$ [95\% CI, 1.05-1.35], $p=$ 0.007 , eTable 3 online).

Table 1 Prevalence of Study Medication Use in Patients Within Physician-Patient Pairs in 2015 in Ontario, by Physician Antibiotic Prescribing Intensity

\begin{tabular}{|c|c|c|c|}
\hline \multirow[t]{2}{*}{ Prevalence, $n(\%)$} & \multicolumn{3}{|c|}{ Physician antibiotic prescribing intensity (among physician-patient pairs, $N=128,979$ ) } \\
\hline & Low $(n=31,845)$ & Average $(n=64,368)$ & High $(n=32,766)$ \\
\hline Benzodiazepine & $4600(14.4)$ & $11,581(18.0)$ & $6725(20.5)$ \\
\hline 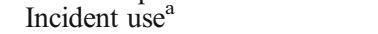 & $1977(6.2)$ & $5653(8.8)$ & $3344(10.2)$ \\
\hline Opioid & $9621(30.2)$ & $21,468(33.4)$ & $12,555(38.3)$ \\
\hline Incident use ${ }^{\mathrm{a}}$ & $4380(13.8)$ & $10,883(16.9)$ & $6389(19.5)$ \\
\hline Proton-pump inhibitor & $9431(29.6)$ & $23,300(36.2)$ & $13,672(41.7)$ \\
\hline Incident use $^{\mathrm{a}}$ & $2817(8.8)$ & $7056(11.0)$ & $4010(12.2)$ \\
\hline \multicolumn{4}{|l|}{ Tracer analysis } \\
\hline Levothyroxine & 5609 (17.6) & $13,204(20.5)$ & 7834 (23.9) \\
\hline 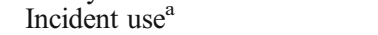 & $1304(4.1)$ & $3299(5.1)$ & $1901(5.8)$ \\
\hline$\beta$-Blocker ophthalmic eye drop & $2231(7.0)$ & $5208(8.1)$ & $2940(9.0)$ \\
\hline Incident use ${ }^{\mathrm{a}}$ & $578(1.8)$ & $1440(2.2)$ & $814(2.5)$ \\
\hline
\end{tabular}

${ }^{a}$ In the year 2015, incident use was determined using a 1-year lookback to ensure that no prescriptions in the prior year were made for the specific medication class 
Table 2 Association between Physician Antibiotic Prescribing Intensity and Likelihood of Prescribing a Study Medication in 2015 to a Patient Within a Physician-Patient Pair in Ontario

\begin{tabular}{|c|c|c|c|c|}
\hline & $\begin{array}{l}\text { Physician antibiotic } \\
\text { prescribing intensity }\end{array}$ & $\begin{array}{l}\text { Unadjusted odds ratio } \\
(95 \% \text { CI })\end{array}$ & $\begin{array}{l}\text { Adjusted odds ratio } \\
(95 \% \mathrm{CI})^{*}\end{array}$ & $p$ value \\
\hline \multirow[t]{3}{*}{ Benzodiazepine } & High & $1.21(1.12-1.32)$ & $1.21(1.11-1.32)$ & \multirow[t]{3}{*}{$<0.001^{\mathrm{a}}$} \\
\hline & Average & 1.00 & 1.00 & \\
\hline & Low & $0.72(0.67-0.78)$ & $0.68(0.63-0.74)$ & \\
\hline \multirow[t]{3}{*}{ Opioid } & High & $1.26(1.17-1.36)$ & $1.28(1.17-1.39)$ & \multirow[t]{3}{*}{$<0.001^{\mathrm{a}}$} \\
\hline & Average & 1.00 & 1.00 & \\
\hline & Low & $0.72(0.67-0.77)$ & $0.67(0.61-0.72)$ & \\
\hline \multirow[t]{3}{*}{ Proton-pump inhibitor } & High & $1.36(1.27-1.47)$ & $1.38(1.27-1.51)$ & \multirow[t]{3}{*}{$<0.001^{\mathrm{a}}$} \\
\hline & Average & 1.00 & 1.00 & \\
\hline & Low & $0.68(0.64-0.73)$ & $0.64(0.59-0.70)$ & \\
\hline \multicolumn{5}{|l|}{ Tracer analysis } \\
\hline \multirow[t]{3}{*}{ Levothyroxine } & High & $1.24(1.17-1.32)$ & $1.23(1.16-1.31)$ & \multirow[t]{3}{*}{$<0.001^{\mathrm{a}}$} \\
\hline & Average & 1.00 & 1.00 & \\
\hline & Low & $0.80(0.75-0.85)$ & $0.80(0.75-0.85)$ & \\
\hline \multirow[t]{3}{*}{$\beta$-Blocker ophthalmic eye drop } & High & $1.11(1.03-1.20)$ & $1.10(1.02-1.19)$ & $0.004^{\mathrm{b}}$ \\
\hline & Average & 1.00 & 1.00 & $0.01^{\mathrm{c}}$ \\
\hline & Low & $0.86(0.79-0.93)$ & $0.86(0.80-0.93)$ & $<0.001^{\mathrm{d}}$ \\
\hline
\end{tabular}

*Adjusted for all individual patient- and physician-level variable listed in eTables 1 and 2

${ }^{a}$ For all unadjusted and adjusted comparisons between high-and average-intensity and low- and average-intensity groups

${ }^{b}$ For unadjusted comparison between high-and average-intensity group

${ }^{c}$ For adjusted comparison between high- and average-intensity group

${ }^{d}$ For unadjusted and adjusted comparisons between low-and average-intensity group

\section{DISCUSSION}

In this study of 1926 physicians caring for older patients in nursing homes, the intensity of a physician's antibiotic prescribing was significantly associated with the likelihood of prescribing benzodiazepines, opioids and proton-pump inhibitors. Being a high-intensity prescriber of antibiotics was associated with a $21 \%, 28 \%$ and $38 \%$ increased risk of prescribing a benzodiazepine, opioid or PPI compared with an average-intensity physician, respectively, after adjusting for individual patient factors. These high-intensity prescribers were also more likely to initiate levothyroxine and $\beta$-blocker eye drops for their patients, and up to six times more likely to prescribe multiple drugs at a higher rate than average-intensity prescribers.

The fact that antibiotic prescribing was associated with prescriptions for our primary study medications in addition to levothyroxine and $\beta$-blocker eye drops suggests that physicians who are high prescribers of certain medications may be high prescribers of all medications. Using a physician's intensity of episodically prescribed medications like antibiotics presents a potentially effective screening method to identify outlier prescribers of multiple medications in large healthcare systems who can then be evaluated on the appropriateness of their prescribing for more than one potentially harmful medication.

We chose to use antibiotics because of their prevalence and large variation in use within nursing homes, supporting the notion that in certain situations, their use may too be potentially inappropriate. ${ }^{14,20,29}$ This variation, along with that of opioids and benzodiazepines, is strongly associated with individual physicians, more so than the characteristics of the patients to whom they are prescribed..$^{20,24,30,34}$ Perhaps most importantly, the episodic nature of antibiotic prescribing allows one to ascribe responsibility to an individual physician and therefore identify the outliers within health systems that may include thousands of physicians and multiple different

Table 3 The Frequency and Likelihood of Highly Prescribing One or More of Benzodiazepines, Opioids and Proton-Pump Inhibitors Across Prescribing Physicians in Ontario 2015, by Physician Antibiotic Prescribing Intensity. We Compared High- with Average-Intensity Antibiotic Prescribers Across the Different Prescriber Scores Based on the Total Number of Medication Classes (Benzodiazepines, Opioids, and PPIs-One Point for Each) in Which They Were a High-Intensity Prescriber. A Score of 0 Served as the Reference for Comparison

\begin{tabular}{|c|c|c|c|c|}
\hline \multirow[t]{2}{*}{$\begin{array}{l}\text { Physician prescribing } \\
\text { score }^{\mathrm{a}}, n(\%)\end{array}$} & \multicolumn{3}{|c|}{$\begin{array}{l}\text { Physician antibiotic prescribing intensity } \\
(N=1926)\end{array}$} & \multirow{2}{*}{$\begin{array}{l}\begin{array}{l}\text { Likelihood of physician } \\
\text { prescribing score }\end{array} \\
\text { Odds ratio }(95 \% \mathrm{CI})\end{array}$} \\
\hline & Low $(n=590)$ & Average $(n=1007)$ & High $(n=329)$ & \\
\hline $\begin{array}{l}0 \\
1 \\
2 \\
3\end{array}$ & $\begin{array}{l}478(81.0) \\
91(15.4) \\
13-17(2.2-2.9)^{\mathrm{d}} \\
4-8(0.7-1.4)^{\mathrm{d}}\end{array}$ & $\begin{array}{l}762(75.7) \\
184(18.3) \\
48(4.8) \\
13(1.3)\end{array}$ & $\begin{array}{l}141(42.9) \\
114(34.7) \\
59(17.9) \\
15(4.6)\end{array}$ & $\begin{array}{l}1.00 \text { (reference) }^{\mathrm{c}} \\
3.35(2.49-4.50)^{\mathrm{c}} \\
6.64(4.36-10.12)^{\mathrm{c}} \\
6.24(2.90-13.39)^{\mathrm{c}}\end{array}$ \\
\hline
\end{tabular}

${ }^{a}$ Physicians are assigned a point for each medication class of benzodiazepines, opioids and PPIs in which they were a high-intensity prescriber, corresponding to a maximum score of 3. Each group is mutually exclusive of another, meaning that a physician cannot be in more than group

${ }^{b}$ Estimated comparing physicians who were high-intensity versus average-intensity antibiotic prescribers for each physician prescribing score with a prescribing score of 0 serving as the reference outcome category

${ }^{c} p<0.001$ for high- compared with average-intensity antibiotic prescribers

${ }^{d}$ Data presented as ranges of values in accordance with IC/ES privacy policy to prevent a disclosure of a cell size containing 5 or less individuals 
medications of interest. Our demonstrated association between antibiotic prescribing and the prescribing of other medications is the key to ensure this strategy may work.

Current initiatives such as the Veteran's Affairs Opioid Safety Initiative ${ }^{1}$ and the Choosing Wisely campaigns focus on avoiding benzodiazepine and opioid use in older adults, ${ }^{2,3}$ and Deprescribing Canada's guideline on use of PPIs, ${ }^{4}$ along with antimicrobial stewardship programs around the world, tends to focus on single drug classes, in part as a way to address the appropriateness of medication decisions. Strategies that target high-volume prescribers of single medication classes appear to be effective in reducing prescribing of those single medications, ${ }^{11}$ but evidence to support similar applications to the prescribing of multiple medication classes is lacking.

Variation in rates of high-risk prescribing for a variety of medications has been used to signal potential quality of care issues, such as in the congressionally mandated Agency for Healthcare Research and Quality's National Healthcare Quality and Disparities Reports in the USA. However, reducing variation is not straightforward and is a persistent challenge. Prior research demonstrated the association between individual physician traits and their prescribing of antibiotics, ${ }^{24}$ suggesting that changing physician behavior to reduce variation might not be impossible using approaches such as audit and feedback. ${ }^{35}$ The use of existing highly developed programs in medication prescribing stewardship ${ }^{36,37}$ presents an exciting opportunity to apply this infrastructure in the context of our findings.

\section{LIMITATIONS}

The generalizability of our findings to the prescribing tendencies of physicians outside the nursing home is not certain and our findings will need to be validated in other care settings. We did not account for potential variations in individual facility practices and medication stewardship in our analytical model, which have been previously demonstrated. ${ }^{19,20}$

We did not capture specific medication-related harm among older adults in this study, although these risks have been well characterized in prior work, such as the risk of respiratory depression, falls and fractures when prescribed opioids or benzodiazepines, ${ }^{6-9}$ and the risk of osteoporotic-related fractures and Clostridium difficile infection with PPIs. ${ }^{10}$

Unexpectedly, receipt of levothyroxine and $\beta$-blocker eye drops was associated with a physician's antibiotic prescribing tendencies. There are two potential explanations for this. Like antibiotics and other medications, the treatment of subclinical hypothyroidism (an elevated thyroid-stimulating hormone with a normal thyroxine (T4) level in an otherwise asymptomatic adult) is variable ${ }^{38}$ and may involve an element of subjective treatment decision-making by the physician. Hence, "high-intensity" prescribers might prescribe all drugs at a higher rate. However, this finding may also reflect residual confounding in unmeasured differences specific to patients, especially if groups of physicians of similar antibiotic prescribing intensity provide care in more than one facility. This effect was minimized through availability of rich clinical and functional individual patient data on the RAI, including frailty, which reflects a patient's overall illness severity to some degree.

Finally, our findings do not explain why certain physicians prescribe differently than their colleagues. Our approach to categorize antibiotic prescribing behavior identifies outliers in prescribing compared with their peers but does not account for the appropriateness of this prescribing behavior. There are many potential factors that motivate a physician's decision to prescribe medications, which may include emotional bias and fear that failing to act will lead to an undesirable outcome, as well as real or perceived demands from patients' families or their caregivers.

\section{CONCLUSIONS}

Physicians who are high prescribers of an individual medication may be a high prescriber of all medications. The association between physician prescribing of episodic medications, such as antibiotics, and their prescribing patterns for more chronic medications such as opioids, benzodiazepines and proton-pump inhibitors offer a novel and potentially useful screening mechanism to identify prescribers more broadly who may benefit from physician-level interventions to optimize overall prescribing in nursing homes.

Acknowledgements: We wish to thank Matthew Kumar for his assistance with the creation of some of the figures. We also thank IMS Brogan Inc. for use of their Drug Information Database.

Corresponding Author: Kieran L. Quinn, MD, MSc; Department of Medicine, Sinai Health System, Toronto, Ontario M5G 1X5, Canada (e-mail: kieran.quinn@sinaihealthsystem.ca).

Funding Information This research was funded in part by the Canadian Frailty Network (SIG2014-M1) which is supported by the Government of Canada through Networks of Centres of Excellence (NCE) Program, and also the Canadian Institutes of Health Research (CIHR) through an operating grant (Exploring frailty and its role in the assessment of high risk medications and risk of poor health outcomes in vulnerable populations-MOP-136854). This study was supported by the Institute for Clinical Evaluative Sciences (ICES), which is funded by an annual grant from the Ontario Ministry of Health and Long-Term Care (MOHLTC). Kieran Quinn receives funding from the CIHR Vanier Scholarship Program, the Eliot Phillipson ClinicianScientist Training Program and the Clinician Investigator Program at the University of Toronto.

Data Availability The dataset from this study is held securely in coded form at the Institute for Clinical Evaluative Sciences (ICES). While data sharing agreements prohibit IC/ES from making the dataset publicly available, access may be granted to those who meet prespecified criteria for confidential access, available at www.ices.on. ca/DAS. The full dataset creation plan and underlying analytic code are available from the authors upon request, understanding that the programs may rely upon coding templates or macros that are unique to ICES. 


\section{Compliance with Ethical Standards:}

Conflict of Interest: The authors declare that they do not have a conflict of interest.

Disclaimer: The opinions, results and conclusions reported in this paper are those of the authors and are independent from the funding sources. No endorsement by IC/ES or the Ontario MOHLTC is intended or should be inferred. Parts of this material are based on data and information compiled and provided by the Canadian Institute for Health Information (CIHI). However, the analyses, conclusions, opinions and statements expressed herein are those of the author, and not necessarily those of CIHI.

Publisher's Note: Springer Nature remains neutral with regard to jurisdictional claims in published maps and institutional affiliations.

\section{REFERENCES}

1. Abbasi J. VHA Initiative Reduces Risky Opioid Prescribing. JAMA. 2017;317(8):797. https://doi.org/10.1001/jama.2017.1087.

2. Choosing Wisely Canada. Opioid Wisely. https://choosingwiselycanada. org/campaign/opioid-wisely/. Published March 1, 2018. Accessed 31 July 2019.

3. Choosing Wisely. American Geriatrics Society. http://www. choosingwisely.org/wp-content/uploads/2013/01/about_ choosingwisely_fivethings.pdf. Published 2013. Accessed 31 July 2019.

4. Farrell B, Pottie K, Thompson W, et al. Deprescribing proton pump inhibitors: Evidence-based clinical practice guideline. Can Fam Physician 2017;63(5):354-364.

5. Rose AJ, Bernson D, Chui KKH, et al. Potentially Inappropriate Opioid Prescribing, Overdose, and Mortality in Massachusetts, 2011-2015. J Gen Intern Med 2018;33(9):1512-1519. https://doi.org/10.1007/ s11606-018-4532-5.

6. Woolcott JC, Richardson KJ, Wiens Mo, et al. Meta-analysis of the impact of 9 medication classes on falls in elderly persons. Arch Intern Med 2009; 169(21):1952-1960. https://doi.org/10.1001/archinternmed. 2009.357.

7. Daoust R, Paquet J, Moore $\mathbf{L}$, et al. Recent opioid use and fall-related injury among older patients with trauma. CMAJ. 2018;190(16):E500E506. https://doi.org/10.1503/cmaj.171286.

8. Finkle WD, Der JS, Greenland $\mathbf{S}$, et al. Risk of fractures requiring hospitalization after an initial prescription for zolpidem, alprazolam, lorazepam, or diazepam in older adults. J Am Geriatr Soc 2011;59(10):1883-1890. https://doi.org/10.1111/j.1532-5415.2011. 03591.x.

9. Vozoris NT, Fischer HD, Wang $\mathbf{X}$, et al. Benzodiazepine use among older adults with chronic obstructive pulmonary disease: a population-based cohort study. Drugs Aging 2013;30(3):183-192. https://doi.org/10. 1007/s40266-013-0056-1.

10. Maes ML, Fixen DR, Linnebur SA Adverse effects of proton-pump inhibitor use in older adults: a review of the evidence. Ther Adv Drug Saf 2017;8(9):273-297. https://doi.org/10.1177/2042098617715381.

11. Hallsworth M, Chadborn T, Sallis A, et al. Provision of social norm feedback to high prescribers of antibiotics in general practice: a pragmatic national randomised controlled trial. Lancet. 2016;387(10029):17431752. https://doi.org/10.1016/S0140-6736(16)00215-4.

12. Chen JH, Humphreys $\mathbf{K}$, Shah NH, Lembke A. Distribution of Opioids by Different Types of Medicare Prescribers. JAMA Intern Med 2016;176(2):259261. https://doi.org/10.1001/jamainternmed.2015.6662.

13. Guthrie B, Donnan PT, Murphy DJ, Makubate B, Dreischulte T. Bad apples or spoiled barrels? Multilevel modelling analysis of variation in high-risk prescribing in Scotland between general practitioners and between the practices they work in. BMJ Open 2015;5(11):e008270. https://doi.org/10.1136/bmjopen-2015-008270.

14. Burke JP. Antibiotic Resistance-Squeezing the Balloon? JAMA. 1998;280(14):1270-1271. https://doi.org/10.1001/jama.280.14.1270.

15. Hunnicutt JN, Chrysanthopoulou SA, Ulbricht CM, Hume AL, Tjia J, Lapane KL. Prevalence of Long-Term Opioid Use in Long-Stay Nursing Home Residents. J Am Geriatr Soc 2018;66(1):48-55. https://doi.org/ $10.1111 /$ jgs. 15080 .
16. Torchia MT, Munson J, Tosteson TD, et al. Patterns of Opioid Use in the 12 Months Following Geriatric Fragility Fractures: A Population-Based Cohort Study. J Am Med Dir Assoc 2019;20(3):298-304. https://doi.org/ 10.1016/j.jamda.2018.09.024.

17. Bourgeois J, Elseviers MM, Azermai M, Van Bortel L, Petrovic M, Vander Stichele RR. Benzodiazepine use in Belgian nursing homes: a closer look into indications and dosages. Eur J Clin Pharmacol 2012;68(5):833-844. https://doi.org/10.1007/s00228-011-1188-z.

18. Helvik A-S, Šaltytè Benth J, Wu B, Engedal K, Selbæk G. Persistent use of psychotropic drugs in nursing home residents in Norway. BMC Geriatr 2017;17(1):52. https://doi.org/10.1186/s12877-017-0440-5.

19. Daneman N, Gruneir A, Newman A, et al. Antibiotic use in long-term care facilities. J Antimicrob Chemother 2011;66(12):2856-2863. https:// doi.org/10.1093/jac/dkr395.

20. Daneman N, Campitelli MA, Giannakeas V, et al. Influences on the start, selection and duration of treatment with antibiotics in long-term care facilities. CMAJ. 2017;189(25):E851-E860. https://doi.org/10. 1503/cmaj.161437.

21. Guinn KL, Macdonald EM, Gomes T, et al. Macrolides, Digoxin Toxicity and the Risk of Sudden Death: A Population-Based Study. Drug Saf 2017;30(2):1114. https://doi.org/10.1007/s40264-017-0539-9.

22. Guinn KL, Macdonald EM, Mamdani MM, Diong C, Juurlink DN, Canadian Drug Safety and Effectiveness Research Network (CDSERN). Lipophilic Statins and the Risk of Intracranial Hemorrhage Following Ischemic Stroke: A Population-Based Study. Drug Saf 2017;40(10):887893. https://doi.org/10.1007/s40264-017-0552-z.

23. Daneman N, Bronskill SE, Gruneir A, et al. Variability in Antibiotic Use Across Nursing Homes and the Risk of Antibiotic-Related Adverse Outcomes for Individual Residents. JAMA Intern Med 2015;175(8): 1331-1339. https://doi.org/10.1001/jamainternmed.2015. 2770.

24. Daneman N, Gruneir A, Bronskill SE, et al. Prolonged antibiotic treatment in long-term care: role of the prescriber. JAMA Intern Med 2013;173(8):673-682. https://doi.org/10.1001/jamainternmed.2013. 3029.

25. Maclagan LC, Maxwell CJ, Gandhi S, et al. Frailty and Potentially Inappropriate Medication Use at Nursing Home Transition. J Am Geriatr Soc 2017;65(10):2205-2212. https://doi.org/10.1111/jgs.15016.

26. Campitelli MA, Maxwell CJ, Giannakeas V, et al. The Variation of Statin Use Among Nursing Home Residents and Physicians: A Cross-Sectional Analysis. J Am Geriatr Soc 2017;65(9):2044-2051. https://doi.org/10. 1111 /jgs. 15013.

27. Mor V. A comprehensive clinical assessment tool to inform policy and practice: applications of the minimum data set. Med Care 2004;42(4 Suppl):III50-III59.

28. Spiegelhalter DJ. Funnel plots for comparing institutional performance. Stat Med 2005;24(8):1185-1202. https://doi.org/10.1002/sim.1970.

29. By the American Geriatrics Society 2015 Beers Criteria Update Expert Panel. American Geriatrics Society 2015 Updated Beers Criteria for Potentially Inappropriate Medication Use in Older Adults. J Am Geriatr Soc. 2015;63(11):2227-2246. https://doi.org/10.1111/jgs.13702.

30. Barnett ML, Olenski AR, Jena AB. Opioid-Prescribing Patterns of Emergency Physicians and Risk of Long-Term Use. N Engl J Med 2017;376(7):663-673. https://doi.org/10.1056/NEJMsa1610524.

31. Bell CM, Bajcar J, Bierman AS, Li P, Mamdani MM, Urbach DR. Potentially unintended discontinuation of long-term medication use after elective surgical procedures. Arch Intern Med 2006;166(22):2525-2531. https://doi.org/10.1001/archinte.166.22.2525.

32. Campitelli MA, Bronskill SE, Hogan DB, et al. The prevalence and health consequences of frailty in a population-based older home care cohort: a comparison of different measures. BMC Geriatr 2016; 16(1): 133. https://doi.org/10.1186/s12877-016-0309-z.

33. Hogan DB, Freiheit EA, Strain LA, et al. Comparing frailty measures in their ability to predict adverse outcome among older residents of assisted living. BMC Geriatr 2012;12(1):56. https://doi.org/10.1186/1471-2318-12-56.

34. Chang H-Y, Murimi IB, Jones CM, Alexander GC. Relationship between high-risk patients receiving prescription opioids and high-volume opioid prescribers. Addiction. 2017. https://doi.org/10.1111/add.14068.

35. Ivers $\mathbf{N}$, Jamtvedt G, Flottorp $\mathbf{S}$, et al. Audit and feedback: effects on professional practice and healthcare outcomes. Cochrane Effective Practice and Organisation of Care Group, ed. Cochrane Database Syst Rev 2012;154(11):669-229. https://doi.org/10.1002/14651858. CD000259.pub3. 
36. Morrill HJ, Caffrey AR, Jump RLP, Dosa D, LaPlante KL. Antimicrobial Stewardship in Long-Term Care Facilities: A Call to Action. J Am Med Dir Assoc. 2016;17(2):183.e1-.e16. https://doi.org/10.1016/j.jamda.2015. 11.013.

37. Livorsi DJ, Heintz B, Jacob JT, Krein SL, Morgan DJ, Perencevich EN. Audit and Feedback Processes Among Antimicrobial Stewardship Programs: A Survey of the Society for Healthcare Epidemiology of America
Research Network. Infect Control Hosp Epidemiol 2016;37(6):704-706. https://doi.org/10.1017/ice.2016.57.

38. Razvi S, Weaver JU, Butler TJ, Pearce SHS. Levothyroxine Treatment of Subclinical Hypothyroidism, Fatal and Nonfatal Cardiovascular Events, and Mortality. Arch Intern Med 2012;172(10):1-7. https://doi.org/10. 1001/archinternmed.2012.1159.

Publisher's Note Springer Nature remains neutral with regard to jurisdictional claims in published maps and institutional affiliations. 